\title{
Priapism induced by use of tamsulosin: A case report and review of the literature
}

\author{
Marcelo Marconi ${ }^{1}$, Pablo Pavez ${ }^{2}$, Ignacio San Francisco ${ }^{2}$, Paulette Narvaez ${ }^{3}$ \\ ${ }^{1}$ Andrology Unit, Department of Urology, Pontificia Universidad Católica de Chile, Santiago, Chile; \\ ${ }^{2}$ Department of Urology, Pontificia Universidad Católica de Chile, Santiago, Chile; \\ ${ }^{3}$ Department of Urology, Hospital Dipreca, Santiago, Chile.
}

\begin{abstract}
Summary Numerous medications have been associated to the development of priapism as an adverse reaction, the most common are intracavernosal vasoactive agents, antipsychotics and antidepressants. Alpha blockers, in particular tamsulosin which is widely used in different urological conditions, has been associated to priapism in only few case reports.

We present the case of a healthy 45-year-old man who medicated himself with two doses of $0.4 \mathrm{mg}$ of tamsulosin due to a renal colic with spontaneous passage of a $3 \mathrm{~mm}$ stone. Eight hours after the second tamsulosin dose the patient developed a persistent painful erection not associated to sexual stimulation that lasted for 6 hours. He was admitted to the emergency room, and after history taking and physical evaluation the diagnosis of ischemic priapism was made. The patient denied consumption of any other medication or drug during the last month, blood tests in particular hemogram were normal and no recent history of pelvic trauma was reported. To achieve detumescence, five boluses of $200 \mathrm{mcg}$ of phenylephrine were injected directly in the corpora cavernosa, no further procedures were needed. In the follow-up the patient had no new priapism episodes and he reported no problems with erections in sexual intercourse. Tamsulosin is one of most indicated medications in urological general practice; though priapism has been rarely associated to its consumption the risk of this side effect exists, suggesting that patients should be counselled about it.
\end{abstract}

KEY WORDS: Priapism; Tamsulosin; Adverse effect.

Submitted 24 April 2019; Accepted 6 May 2019

\section{INTRODUCTION}

Priapism is defined has a prolonged painful erection lasting for more than 4 hours in the absence of sexual stimulation and remaining despite orgasm (1). The three main subtypes are ischemic, non-ischemic and stuttering, being ischemic priapism the most common form, characterized by absence of intracavernous arterial inflow. The incidence of priapism in the general population is low (0.5-0.9 cases per 100,000 person-years); however, it is considered a medical emergency and should be treated promptly to avoid further complications, in particular corporal fibrosis and erectile dysfunction.

Although a specific etiology cannot be found in up to one-third of the cases of ischemic priapism, defined conditions such as blood dyscrasias and neurological disorders have been associated to its development. Priapism can also develop as a side effect of different medications and drugs, being the most common vasoactive intracavernosal injections, psychotropic medications and recreational drugs (i.e. alcohol, cocaine) (2). Alpha-blockers such as prazosin, terazosin and doxazosin have also been reported as an etiology of priapism. Tamsulosin, an alpha la blocker, has rarely been related to priapism; however, is one of the most indicated medications in general urological practice being the first therapeutic line for the management of benign prostatic hyperplasia (3) and treatment of distal ureteral stones (4). The objective of this case report is to present a case of tamsulosin induced ischemic priapism.

\section{Case presentation}

A healthy 45-year-old man with history of one renal colic episode with spontaneous stone elimination 12 months earlier, develops forty-eight hours before consultation a colic left flank pain and lower urinary tract symptoms, in particular urgency and increased urinary frequency, with no fever or macroscopic hematuria. The patient interpreted himself as developing a new renal colic episode so auto-medicated with ketorolac $10 \mathrm{mg}$ three times a day and tamsulosin $0.4 \mathrm{mg}$ once a day. The patient had no history of diabetes, hypertension, neurologic diseases, hematologic disease or any type of drug consumption or abuse. Thirty-six hours after the first symptoms and after two doses of tamsulosin the patient spontaneously eliminated a $3 \mathrm{~mm}$ stone with no other incidents. The night before consultation approximately eight hours after stone elimination the patient woke up at $6 \mathrm{AM}$ with his usual morning erection, he urinated with no difficulties but the erection persisted during the following 5 hours without any sexual stimulation at that time or the night before. The patient tried to achieve detumescence by walking and local ice application with no success at home. He was admitted at noon with already six hours of a constant, painful and unrelated to sexual stimuli erection.

At admission, the patient was evaluated by the emergency room physician, who collected a completed medical history excluding consumption of drugs (cocaine, marijuana), antidepressants, antipsychotics, narcotics, intracavernosal injection of vaso-active agents, phosphodiesterase 5 inhibitors, or any other substance associated 
with priapism risk. The patient had no history of pelvic trauma either major or minor. The only drugs the patient had consumed in the last 48 hours were five $10 \mathrm{mg} \mathrm{sep}$ arated doses of ketorolac and two $0.4 \mathrm{mg}$ doses of tamsulosin. He had a visual analog visual for pain (range 010) of 7 secondary to the painful erection.

At admission the physical examination showed: temperature $36,6^{\circ} \mathrm{C}$, pulse $84 /$ minute, respiratory rate $18 /$ minute and blood pressure 137/88. Heart, lung, abdominal and neurologic examination were normal. Genital examination revealed no skin lesions, painless testicular examination with normal volume and a complete painful rigid erection with no tumescence of the glans. A urine analysis revealed microscopic hematuria with no other pathological signs, urine culture developed no bacterial growth 48 hours later, and hemogram was normal with no signs of hematologic disease. After evaluation, a consultation for urologic evaluation was asked. After history taking and physical evaluation, the urologist confirmed the diagnosis of ischemic priapism.

To achieve detumescence, after administration of local anesthesia to the base of the penile shaft and while monitoring the heart rate and blood pressure of the patient, boluses of 200 microgram phenylephrine in a two milliliter solution were injected directly in the corpora cavernosa. After five boluses (1000 micrograms) and 20 minutes, detumescence was achieved. No further procedures were needed. The patient was send home with the indication to avoid tamsulosin intake and sexual intercourse for the next seven days. In the follow-up the patient had no new priapism episodes and reported no problems with erections and sexual intercourse.

\section{Discussion}

Adverse events to different type of medications is one of the most important causes of priapism. The most common drugs involved in priapism development are vasoactive intracavernosal injections, anti-psychotics, antidepressants, cocaine, alcohol (Table 1). There are few published reports associating the consumption of alphablockers to the development of priapism, in particular tamsulosin. In a previous systematic review, 13 articles reported a cause-effect relation, among which only three cases were secondary to tamsulosin (5). In one of those cases, tamsulosin was associated to a partial thrombosis of the corpora cavernosa (6), and in a second case the association of tamsulosin to a drug (Boceprevir-CYP3A4) that inhibits its degradation triggered a priapism episode (7). When analyzing our case, we were not able to find any other etiology of priapism in our patient. He was healthy, with no hematological or neurologic diseases, and the only two medications he had consumed in the last 48 hours were ketorolac and tamsulosin. There are no reports in the literature associating ketorolac with the development of priapism. As an alpha-blocker tamsulosin would interfere with the detumescence mechanism that is mediated by adrenaline and nor-adrenaline acting on alpha-adrenergic receptors in the corpora cavernosa. Considering the previous case-reports and ours, it seems that the development of priapism would be independent of the number tamsulosin doses.
Table 1.

Drugs associated to the development of priapism.

\begin{tabular}{l} 
Intracavernosal vaso-active drugs \\
\hline Prostaglandin - Papaverine - Phentolamine \\
\hline Antipsychotics \\
\hline Clozapine - Olanzapine - Risperidone - Chlorpromazin - Haloperidol - \\
Thoridazine \\
\hline Antidepressants \\
\hline Trazodone - Imipramine - Bupropion - Fluoxetine - Lithium \\
\hline Antianxiety agents \\
\hline Hydroxyzine \\
\hline Antihypertensives \\
\hline Ntiroglicerine - Hydralazine - Guanethidine - Propanolol - Verapamil \\
\hline Alpla-blockers \\
\hline Doxazosine - Prazosine - Terazosin - Tamsulosin \\
\hline Theophylline \\
\hline Vancomycin \\
\hline Heparin - Warfarin \\
\hline Erythropoietin \\
\hline Alcohol \\
\hline Cocaine \\
\hline Cannabis \\
\hline
\end{tabular}

Even though, tamsulosin related priapism has been rarely reported; the association is relevant considering that this medication is commonly prescribed by urologists to treat benign prostatic hyperplasia, which is an extremely frequent condition in general population. When prescribing tamsulosin, priapism is almost never mentioned as a potential side-effect, questionable our report together with previous ones suggest that patients should be counselled.

\section{Conclusions}

Tamsulosin is one of most prescribed medications in urological practice, even though priapism has been rarely associated to its consumption, the risk exists, suggesting that patients should be counselled about it.

\section{AUTHORS CONTRIBUTION}

$\mathrm{MM}$ and FP contributed to the writing of the first draft of the report and the initial discussion. MM was involved in the care and therapy of the patient. ISF and PN reviewed the manuscript. MM is the guarantor of this work and, as such, had full access to all the data in the study and takes responsibility for the integrity of the data and the accuracy of the data analysis. All authors read and approved the final manuscript.

\section{References}

1. Salonia A, Eardley I, Giuliano F, et al. European Association of Urology guidelines on priapism. Eur Urol. 2014; 65:480-489

2. Muneer, A. Comparison of EAU and UK guidelines on priapism. Journal of Clinical Urology. 2017; 11:127-131. 
3. Foster HE, Barry MJ, Dahm P, et al. Surgical Management of Lower Urinary Tract Symptoms Attributed to Benign Prostatic Hyperplasia: AUA Guideline. J Urol. 2018; 200:612-619.

4. Turk C, Neisius AP, Seitz C,, et al. (2018) EAU guidelines on urolithiasis. https://uroweb.org/guideline/urolithiasis. Accessed 04 April 2019

5. Spagnul SJT, et al. Adrenergic a-blockers: an infrequent and over- looked cause of priapism. International Journal of Impotence Research. 2011; 23:95-98.

6. Kilinc M, Piskin S, Guven R, et al. Partial priapism secondary to tamsulosin: a case report and review of the literature. Andrologia. 2009; 21:199-201.

7. Hammond KP, Nielsen C, Linnebur SA, et al. Priapism induced by boceprevir-CYP3A4 inhibition and $\alpha$-adrenergic blockade: case report. Clin Infect Dis. 2014; 58:e35-8.

\section{Correspondence}

Marcelo Marconi, MD (Corresponding Author)

mmarconi@andro.cl

Andrology Unit, Department of Urology, Pontificia Universidad Catolica de Chile, Cruz del Sur 177, Santiago (Chile)

Pablo Pavez, MD

info@andro.cl

Ignacio San Francisco, MD

isanfrancisco@med.puc.cl

Department of Urology, Pontificia Universidad Católica de Chile,

Santiago (Chile)

Paulette Narvaez, MD

kikinarvaez@gmail.com

Department of Urology, Hospital Dipreca, Santiago (Chile) 